\title{
The intriguing relationship between epilepsy and type 1 diabetes mellitus. Reply to Sander JW, Novy J, Keezer MR [letter]
}

\author{
I-Ching Chou ${ }^{1,2} \cdot$ Chia-Hung Kao ${ }^{3}$
}

Received: 26 April 2016/Accepted: 28 April 2016/Published online: 18 May 2016

(C) Springer-Verlag Berlin Heidelberg 2016

Keywords Epilepsy · National Health Insurance Research Database · Type 1 diabetes

\begin{abstract}
Abbreviations
ADHD Attention deficit hyperactivity disorder

NHI (Taiwan) National Health Insurance (programme)

NHIRD (Taiwan) National Health Insurance Research Database

CNS Central nervous system
\end{abstract}

To the Editor: We thank Sander et al for their letter [1] commenting on our recent article [2], in which we reported that patients with type 1 diabetes mellitus are at an increased risk of developing epilepsy. Sander et al point out the strengths and challenges of the study. First, our researchers conducted a retrospective population-based cohort study using claims data from the Taiwan National Health Insurance Research Database (NHIRD). The coverage rate of the Taiwan National Health Insurance programme (NHI) reached $99 \%$ of the 23 million residents of Taiwan in 1998. The NHIRD is renewed annually

Chia-Hung Kao

d10040@mail.cmuh.org.tw

1 Department of Pediatric Neurology, China Medical University Children's Hospital, Taichung, Taiwan

2 Graduate Institute of Integrated Medicine, College of Chinese Medicine, China Medical University, Taichung, Taiwan

3 Graduate Institute of Clinical Medical Science and School of Medicine, College of Medicine, China Medical University, No. 2, Yuh-Der Road, Taichung 40447, Taiwan and includes registries for beneficiaries, disease diagnosis records, medical prescriptions and other medical services. It is a large, population-based sample effectively including all Taiwanese residents. The registries of NHI claims primarily serve the purpose of administrative billing and do not undergo verification for scientific purposes. Therefore, we were unable to contact the patients directly to obtain more information, because of the anonymity assured by the identification numbers.

Second, metabolic abnormalities of type 1 diabetes, such as hyper- and hypoglycaemia, may have a damaging effect on the central nervous system (CNS) and be associated with significant long-term neurological sequelae. Sander et al [1] point out that our data included hypoglycaemia as a potential confounder, although hyperglycaemia is not considered in the statistical analysis. This was because we used an in-hospital diagnosis (ICD-9-CM [www.icd9data.com/2007/Volume1] 251.0-251.2 and 775.6), whereby a patient was deemed to have hypoglycaemia occurring during follow-up. As suggested by Sander et al, patients with hyperglycaemia might have been underestimated, because this may not translate into a recoded diagnosis.

Third, we found that younger age was associated with an increased risk of developing epilepsy; those aged at least 6 years were 40 percent less likely to have epilepsy than those under 6 years. This is consistent with previous studies indicating that young age is an important risk factor for electroencephalographic abnormalities [3].

Fourth, 59 people were reported to have 'developed' epilepsy over the course of the study, but $27 \%$ of these (16 of 59) had a prior history of epilepsy, which would generally make these prevalent rather than incident cases. Actually, epilepsy is characterised by spontaneous recurrent seizures, but can be a pervasive condition of which seizures are just one expression [4]. Several mechanisms explain how epilepsy and comorbidities are associated, including shared risk factors and bidirectional 
relations [5]. In our previous studies, we used the NHIRD to examine similar relationships between epilepsy and a number of conditions, including schizophrenia and attention deficit hyperactivity disorder (ADHD) $[6,7]$. We found a strong bidirectional relationship between schizophrenia and epilepsy. The incidence of epilepsy was 5.88 times higher in the schizophrenia cohort than in the non-schizophrenia cohort. The incidence of schizophrenia was 7.65 times higher in the epilepsy cohort than in the non-epilepsy comparison cohort [6]. In another study, we found that the possibility of developing ADHD among epileptic patients is higher (adjusted HR 2.54). Conversely, ADHD increases the risk of subsequent epilepsy (adjusted HR 3.94), and vice versa [7]. Such a relationship may be explained by the existence of common pathogenic mechanisms in both conditions, including changes in neuroanatomy, neural migration dysfunction, genetic susceptibility or environmental factors.

Further studies are needed to clarify the mechanism of the particular relationship between type 1 diabetes and epilepsy. In our review, we present four hypotheses to explain this relationship: metabolic abnormalities, cerebrovascular disease, genetic factors and autoimmune-related factors. Autoantibodies associated with epilepsy may be a new window for future research, because several CNS disorders associated with specific antibodies to ion channels, receptors and other synaptic proteins have been recognised [8]. Among the growing list of neuronal autoantibodies identified in a subgroup of patients with epilepsy, some may appear to play a pathogenic role.

Identifying these mechanisms is an important consideration in the clinical management of epilepsy, and the causative factors between type 1 diabetes and the increased risk of epilepsy require further investigation. We hope our results could provide evidence to help improve the prognosis of children with type 1 diabetes.

Funding This study was supported in part by the study projects (DMR105-032) in our hospital; Taiwan Ministry of Health and Welfare Clinical Trial and Research Center of Excellence (MOHW105-TDU-B-212133019); China Medical University Hospital, Academia Sinica Taiwan
Biobank, Stroke Biosignature Project (BM10501010037); NRPB Stroke Clinical Trial Consortium (MOST 104-2325-B-039-005); Tseng-Lien Lin Foundation, Taichung, Taiwan; Taiwan Brain Disease Foundation, Taipei, Taiwan; Katsuzo and Kiyo Aoshima Memorial Funds, Japan; and China Medical University (CMU) under the Aim for the Top University Plan of the Ministry of Education, Taiwan. The funders had no role in the study design, data collection and analysis, decision to publish or preparation of the manuscript. No additional external funding was received for this study.

Duality of interest The authors declare that there is no duality of interest associated with this manuscript.

Contribution statement All authors have contributed substantially to, and are in agreement with, the content of the manuscript.

\section{References}

1. Sander JW, Novy J, Keezer MR (2016) The intriguing relationship between epilepsy and type 1 diabetes mellitus. Diabetologia. doi:10.1007/s00125-016-3982-8

2. Chou IC, Wang CH, Lin WD, Tsai FJ, Lin CC, Kao CH (2016) Risk of epilepsy in type 1 diabetes mellitus: a population-based cohort study. Diabetologia. doi:10.1007/s00125-016-3929-0

3. Soltész G, Acsádi G (1989) Association between diabetes, severe hypo-glycaemia, and electroencephalographic abnormalities. Arch Dis Child 64:992-996

4. Aicardi J (1996) Epilepsy as a non-paroxysmal disorder. Acta Neuropediatrica 2:249-257

5. Keezer MR, Sisodiya SM, Sander JW (2016) Comorbidities of epilepsy: current concepts and future perspectives. Lancet Neurol 15: 106-115

6. Chang YT, Chen PC, Tsai IJ et al (2011) Bidirectional relation between schizophrenia and epilepsy: a population-based retrospective cohort study. Epilepsia 52:2036-2042

7. Chou IC, Chang YT, Chin ZN et al (2013) Correlation between epilepsy and attention deficit hyperactivity disorder: a populationbased cohort study. PLoS One 8:e57926

8. Vincent A, Bien CG, Irani SR, Waters P (2011) Autoantibodies associated with diseases of the CNS: new developments and future challenges. Lancet Neurol 10:759-772 